\title{
ASTEROSEISMOLOGY OF THE $\beta$ CEPHEI STAR 12 (DD) LACERTAE
}

\author{
W.A. DZIEMBOWSKI \\ Copernicus Astronomical Center \\ ul. Bartycka 18, 00-716 Warsaw, Poland \\ AND \\ M. JERZYKIEWICZ \\ Wroctaw University Observatory \\ ul. Kopernika 11, 51-622 Wroctaw, Poland
}

\section{The observed frequencies}

The frequency spectrum in Fig. 1 shows that at least five pulsation modes are excited in DD Lac. Three frequencies, $f_{1}, f_{4}$, and $f_{3}$, form an equidistant triplet. In addition to the value of the central frequency, $f_{4}$, the triplet can be characterized by the mean separation, $S=\left(f_{3}-f_{1}\right) / 2$, and the asymmetry, $\Delta f=f_{4}-\left(f_{1}+f_{3}\right) / 2$. Taking the values of the frequencies from a recent analysis of all available data (Pigulski 1994), we get $S=0.15544 \pm 0.00021$ and $\Delta f=-0.00014 \pm 0.00029 \mathrm{~d}^{-1}$.

\section{The discrepant spherical harmonic degrees}

For the $f_{1}$ mode, the $l$ value has been derived from the observed light, color and radial-velocity amplitudes by Cugier et al. (1994). These authors showed that modes with different $l \leq 2$ are particularly well resolved when the color to light amplitude ratio, $A_{U-V} / A_{V}$, is used as abscissa, and the radial-velocity to light amplitude ratio, $K / A_{V}$, as ordinate. In such a diagram, the $f_{1}$ term falls on the $l=1$ sequence.

For the $f_{2}$ mode, the $U B V$ photometry of Sato $(1973,1977,1979)$ and all available radial velocities (Pigulski 1994) yield $A_{U-V} / A_{V}=0.30 \pm 0.10$ and $K / A_{V}=460 \pm 35$ $\mathrm{km} \mathrm{s}^{-1} / \mathrm{mag}$. In the above-mentioned diagram of Cugier et al. (1994) these coordinates indicate $l=1$ or 2 .

The above identifications can be compared with the results of the line-profile observations. Unfortunately, the three modern line-profile studies of DD Lac yield conflicting results. Smith (1980) maintains that the $f_{1}, f_{4}, f_{3}$ triplet should be identified with the $l=2, m=0,-1,-2$ states, and that the $f_{2}$ mode is radial. On the other hand, Mathias et al. (1994) conclude that the $f_{1}$ mode is either sectoral, or tesseral with $l-1=|m|$. More recently, the line-profile data of Mathias et al. (1994) have been re-analyzed by Aerts (1996). She finds $l=2, m=-1$ for $f_{1}$, and $l=2, m=-2$ 


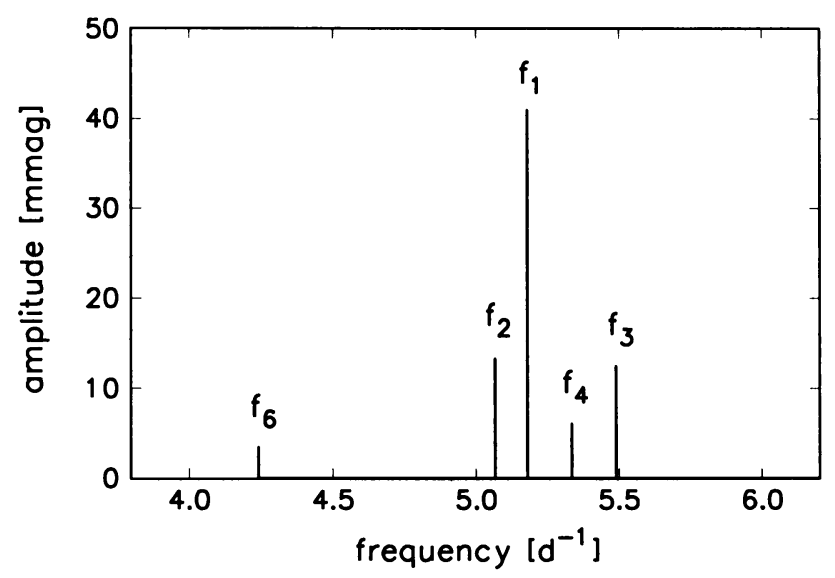

Figure 1. The frequency spectrum of DD Lac, derived by Jerzykiewicz (1978) from the yellow-magnitude data of Abrami et al. (1957). The combination frequency $f_{5}=f_{1}+f_{4}$ is not shown.

for $f_{3}$, but $l=3, m=+1$ for $f_{4}$. Thus, according to Aerts (1996), the triplet $f_{1}, f_{4}$, $f_{3}$ does not consist of three $m$ states of the same $l$. For $f_{2}$, Aerts (1996) gets $l=2$ or 3 and $m=0$.

The only constraint which follows from these discrepant results is that the $f_{1}$ mode is nonradial, with $l$ equal to either 1 or 2 .

\section{The effective temperature and surface gravity of DD Lac}

From the star's MK spectral type of B 1.5 III and the OAO-2 empirical effective temperatures (Code et al. 1976), we derived $\log T_{\text {eff }}=4.369$. Another value can be obtained from the Strömgren indices. Using the observed $c_{0}$ and $\beta$, and the recent calibration of Napiwotzki et al. (1993), we got $\log T_{\text {eff }}=4.380$. The mean of these two numbers, 4.374 , we adopt as $\log T_{\text {eff }}$ of DD Lac. Taking into account uncertainties of the calibrations and the mean errors of the data, we estimate the standard deviation of this value to be 0.020 .

We also derived $\log g$ of DD Lac. Using the observed value of $\beta$ and Smalley and Dworetsky's (1995) grid of synthetic $\beta$ indices, we obtained $\log g=3.76 \pm 0.15$.

In the $\log T_{\text {eff }}-\log g$ plane, the values we obtained place DD Lac close to the 12 $M_{\odot}$ evolutionary track, at $\log L / L_{\odot}=4.3$, that is, in the advanced phases of core hydrogen-burning. The masses allowed by the mean error of $\log T_{\text {eff }}$ span the range from 10 to $13.5 M_{\odot}$.

\section{The triplet}

We shall now assume that $f_{1}, f_{4}$, and $f_{3}$ arise as a result of rotational splitting of an $l>0$ mode. According to the conclusion reached at the end of Sect. 2, the $l$ in question must be 1 or 2 . Since for a given $l$ there are $2 l+1$ spherical harmonic orders, $m$, there is only one possibility of accounting for the frequency triplet if $l=1$, and four possibilities if $l=2$. If $l=1$, the order of frequencies implies the following $m$ - 


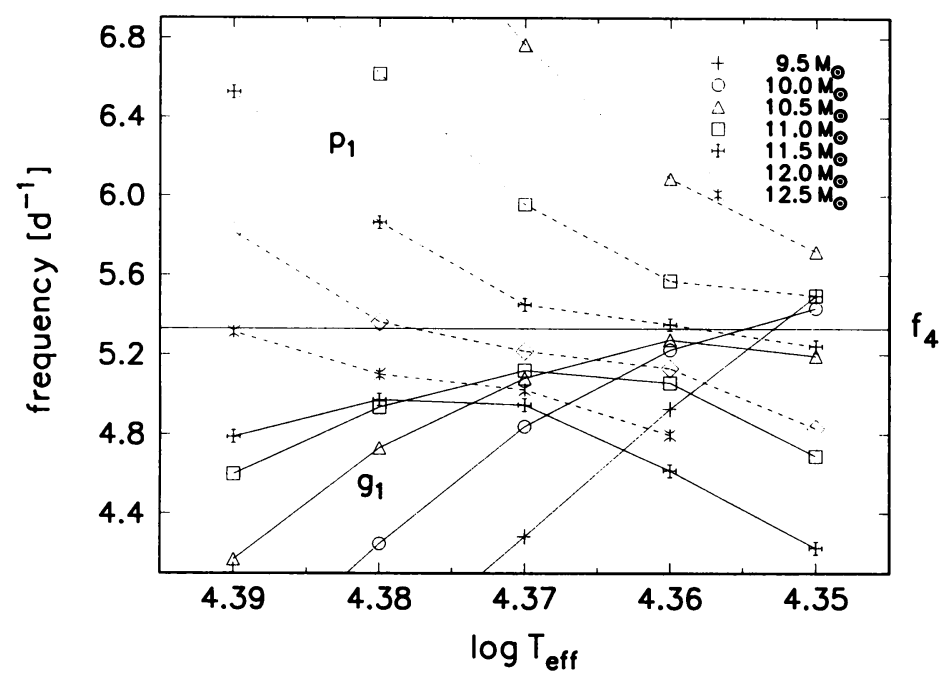

Figure 2. Fitting the observed $f_{4}=5.33427 \mathrm{~d}^{-1}$ to the $l=1, m=0$ modes.

triplet: $m=+1$ (retrograde) for $f_{1}, 0$ for $f_{4}$, and -1 (prograde) for $f_{3}$. If $l=2$, the four possible $m$-triplets are: $(0,-1,-2),(1,0,-1),(2,1,0)$, and $(2,0,-2)$.

\section{The radial order of the $f_{4}$ mode}

A number of modes of $l=1$ or 2 , and $m=-1,0$ or 1 , with frequencies close to $f_{4}$ are unstable in models of DD Lac in the range of effective temperature and mass allowed by the position of the star in the $\log T_{\text {eff }}-\log g$ plane. An example is presented in Fig. 2, where computed frequencies of the two $l=1, m=0$ modes are plotted together with $f_{4}$ (horizontal solid line). The designations $p_{1}$ and $g_{1}$ refer to properties of the modes on ZAMS, where the $p$-and $g$-mode spectra are separated in frequency. The models were computed with the updated OPAL opacities (Iglesias and Rogers 1996) for $X=0.7$ and $Z=0.02$.

As can be seen from Fig. $2, f_{4}$ can be fitted with the frequency of the $p_{1}$ mode in the whole range of $\log T_{\text {eff }}$. On the other hand, for the $g_{1}$ mode such fit is possible only at the lower limit of the temperature range and for the lowest-mass models which have $\log g$ still consistent with the observed one. In addition, an $l=1, m=0, p_{2}$ mode in models with $\log T_{\text {eff }} \approx 4.39$ and $M>13 M_{\odot}$ (not shown in Fig. 2) also has its frequency close to $f_{4}$.

\section{The mean separation and asymmetry of the triplet}

Having identified the modes that could reproduce $f_{4}$ (those shown in Fig. 2 and many others), we varied the rotation frequency, $\Omega$, until computed frequencies of the outlying members of the triplet, $f_{1}$ and $f_{3}$, were also fitted. The rule we adopted was that the observed mean separation of the triplet, $\mathrm{S}$, should be reproduced exactly. The interaction between rotation and pulsation was treated as in Soufi et al. (1997). However, only terms up to quadratic ones in rotation frequency were kept in the 
perturbation treatment. The effect of the near degeneracy of modes differing in $l$ by 2 was taken into account.

Out of a large number of models computed in this way, we selected those which had the smallest asymmetry, $\Delta f$, and reproduced - at least approximately - the two frequencies outside the triplet, $f_{2}$ and $f_{6}$. In all cases we encountered the following two problems: (1) the number of unstable modes was much greater than observed; this problem is common to all linear pulsation analyses (see, e.g., Dziembowski 1997), and (2) the computed $\Delta f$ was greater than the observed one. A discussion of three representative models has been already provided by one of us (WAD, this volume).

This work was supported by KBN grants 2 P30401307 and 2 P03D 01611.

\section{References}

Abrami, A., Bakos, G., Broglia, P. et al., 1957, Nature, 180, 1112

Aerts, C., 1996, Astron. Astrophys., 314, 115

Code, A.D., Davis, J., Bless, R.C., and Hanbury Brown, R., 1976, Astrophys. J., 203, 417

Cugier, H., Dziembowski, W.A., and Pamyatnykh, A.A., 1994, Astron. Astrophys., 291, 143

Dziembowski, W.A., 1997, in J. Provost and F.X. Schmider (eds) IAU Symposium No. 181 - Sounding Solar and Stellar Interiors, in press

Iglesias, C.A. and Rogers, F.J., 1996, Astrophys. J., 464, 943

Jerzykiewicz, M., 1978, Acta Astronomica, 28, 465

Mathias, P., Aerts, C., Gillet, D., and Waelkens, C., 1994, Astron. Astrophys., 289, 875

Napiwotzki, R., Schönberner, D., and Wenske, V., 1993, Astron. Astrophys., 268, 653

Pigulski, A., 1994, Astron. Astrophys., 292, 183

Sato, N., 1973, Astrophys. Space Sci., 24, 215

Sato, N., 1977, Astrophys. Space Sci., 48, 453

Sato, N., 1979, Astrophys. Space Sci., 66, 309

Smalley, B. and Dworetsky, M.M., 1995, Astron. Astrophys., 293, 446

Smith, M.A., 1980, Astrophys. J., 240, 149

Soufi, F., Goupil, M.J., and Dziembowski, W.A., 1997, Astron. Astrophys., in press 